\title{
A Bayesian analysis of small $n$ sequential multiple assignment randomized trials (snSMARTs)
}

\author{
Boxian Wei1ㅇ | Thomas M. Braun ${ }^{1}$ | Roy N. Tamura ${ }^{2} \mid$ Kelley M. Kidwell ${ }^{1}$
}

${ }^{1}$ Department of Biostatistics, School of Public Health, University of Michigan, Ann Arbor, Michigan

${ }^{2}$ Health Informatics Institute, University of South Florida, Tampa, Florida

\section{Correspondence}

Boxian Wei, Department of Biostatistics, University of Michigan, School of Public Health, Ann Arbor, MI 48109.

Email: boxian@umich.edu

\section{Funding information}

Patient-Centered Outcomes Research Institute (PCORI) Award, Grant/Award Number: ME-1507-31108

\begin{abstract}
Designing clinical trials to study treatments for rare diseases is challenging because of the limited number of available patients. A suggested design is known as the small $\mathrm{n}$ sequential multiple assignment randomized trial (snSMART), in which patients are first randomized to one of multiple treatments (stage 1). Patients who respond to their initial treatment continue the same treatment for another stage, while those who fail to respond are rerandomized to one of the remaining treatments (stage 2). The data from both stages are used to compare the efficacy between treatments. Analysis approaches for snSMARTs are limited, and we propose a Bayesian approach that allows for borrowing of information across both stages. Through simulation, we compare the bias, root-mean-square error, width, and coverage rate of $95 \%$ confidence/credible interval of estimators from of our approach to estimators produced from (i) standard approaches that only use the data from stage 1, and (ii) a log-Poisson model using data from both stages whose parameters are estimated via generalized estimating equations. We demonstrate the root-mean-square error and width of $95 \%$ confidence/credible intervals of our estimators are smaller than the other approaches in realistic settings, so that the collection and use of stage 2 data in snSMARTs provide improved inference for treatments of rare diseases.
\end{abstract}

\section{KEYWORDS}

bias, clinical trial, joint model, mean-square error, rare disease

\section{1 | INTRODUCTION}

The Orphan Drug Act defines rare diseases as disorders affecting fewer than 200000 individuals in the United States. ${ }^{1}$ More than 8000 recognized rare diseases affect almost 30 million individuals and their families in the United States. ${ }^{2}$ Identifying optimal treatment options for patients living with rare diseases is challenging due to the low number of individuals affected. Randomized clinical trials (RCTs) are generally regarded as providing the strongest scientific evidence for the efficacy of a treatment. Randomized clinical trials attempt to minimize bias and balance confounders across treatments by employing randomization. ${ }^{3}$ However, confirmatory RCTs often require a large number of subjects, which is difficult to attain in rare disease trials. Thus, RCTs studying treatment for rare diseases commonly have reduced power compared with studies of nonrare diseases. As a result, rare disease trials are more likely to be single arm (63.0\% versus $29.6 \%$ for nonrare disease trials) and nonrandomized (64.5\% versus $36.1 \%$ for nonrare disease trials). ${ }^{4}$ Small sample trials of rare diseases that are randomized and multiarm are most likely crossover, n-of-1, or adaptive designs. ${ }^{5}$

There are disadvantages of the trial designs currently used in the rare disease landscape. For example, single arm studies are employed when the objective of the trial is to obtain preliminary evidence of the treatment efficacy and to collect 
additional safety data. As a result, single arm trials are not generally used to confirm efficacy. ${ }^{6}$ In a crossover study, all subjects receive all experimental treatments. By design, each subject is their own control so that confounding and between-subject variance is reduced, leading to the need of fewer subjects than a parallel design. However, since all participants receive all candidate treatments by crossover, the treatments may expose participants to additional toxicities than a standard RCT or switch participants from an efficacious treatment to a nonefficacious treatment. These challenges have inspired the design of alternative crossover trials so that recruitment might be enhanced by offering patients the ability to switch treatment if they received no benefit from the first. ${ }^{7}$

An n-of- 1 trial is conducted in a single participant with multiple crossover treatment assignments. In an n-of- 1 trial, a participant is their own control so that confounding is reduced and the data can suggest which treatment produces a satisfactory outcome for the participant. However, an n-of- 1 trial usually requires multiple crossover treatment assignments to defend against the effect of treatment across time, measurement error, and error from the participant's condition differing across time. The multiple crossover treatment assignments potentially prolong the duration of the trial, which may be burdensome for the participant and requires a well-developed trial protocol to keep the participant engaged.

Adaptive designs allow for design parameters, such as the sample size, randomization fraction, population recruited, or doses, to be altered during the trial after interim data evaluation. ${ }^{6}$ The adaptiveness may reduce the number of subjects recruited to inferior treatment, increase efficiency, improve recruitment, and take advantage of accumulating data to enable early stopping of the trial. Alternatively, an adaptive trial is often more complex to design and analyze than other standard clinical trial designs and is susceptible to bias due to temporal drift in participant characteristics.

Although the aforementioned designs described may be useful to study treatments in rare diseases, many have called for more innovative trial designs. ${ }^{5}$ An example of such a design was proposed by Honkanen et al. ${ }^{8}$ This design consists of an initial randomized placebo-controlled stage, a randomized withdrawal stage for subjects who responded, and a third randomized stage for placebo nonresponders who subsequently respond to treatment. As an alternative way for rerandomizing subjects, we propose design and methodological improvements for a small $\mathrm{n}$ sequential multiple assignment randomized trial, the snSMART. ${ }^{9}$

An snSMART is an application of a SMART design ${ }^{10-12}$ in small samples (eg, rare diseases). In sequential multiple assignment randomized trials (SMARTs), and hence snSMARTs, patients may be sequentially randomized to treatments where second-stage treatment depends on response to first-stage treatment. For example, in the ARAMIS (a randomized multicenter study for isolated skin vasculitis trial) trial, a two-stage snSMART design to compare three active treatments (Figure 1), patients are randomized equally to one of the treatment arms and followed for six months. The responders continue the same treatment for another six months, while the nonresponders are rerandomized to one of the remaining treatments for an additional six months. The outcome of interest is a binary indicator of response to treatment as defined by a combination of participant and physician measures.

Although SMARTs and snSMARTs have similar designs, we have differentiated their names because each has a distinctly different inferential goal. In a SMART, often the goal is to identify effective treatment sequences or dynamic treatment regimens that define a personalized treatment guideline for each patient that consists of a first-stage treatment, followed by a second stage treatment. ${ }^{13,14}$ In contrast, the goal of an snSMART here is in finding one superior treatment among several that would be used by itself to treat patients. Thus, in this paper, we are not interested in identifying dynamic treatment regimens within an snSMART.

We also highlight that some existing designs allow for absorbing endpoints, at which point all patients are assigned to a different treatment. ${ }^{7,15}$ In contrast, SMARTs and snSMARTs typically do not deal with absorbing endpoints because a treatment is assigned at each stage sequentially depending on the observed outcome of response at the end of current treatment stage in SMARTs and snSMARTs.

Compared with a traditional multistage design, such as a crossover design, the snSMART is attractive because it allows participants who have a satisfactory outcome from their treatment to continue to receive that treatment and who do not have a satisfactory outcome from the treatment to switch to another treatment. Hence, an snSMART design may help to improve participant recuitment and retention. However, analytic methods for an snSMART are not fully established, so that the efficiency gains of an snSMART design compared with other designs in rare disease research have not yet been confirmed.

Our methods are motivated by the snSMART ARAMIS, the design of which mimics the SMART design in metastatic renal cancer. ${ }^{16,17}$ ARAMIS (NCT02939573) is a multinational trial to evaluate different treatment options for patients with skin vasculitis. Vasculitides are uncommon diseases, which can affect almost any organ; although, vasculitis frequently involves the skin as an isolated process or as part of systemic vasculitis. Without high-quality studies to guide the management of skin vasculitis, treatment decisions are made based on anecdotal experience and expert opinions. 


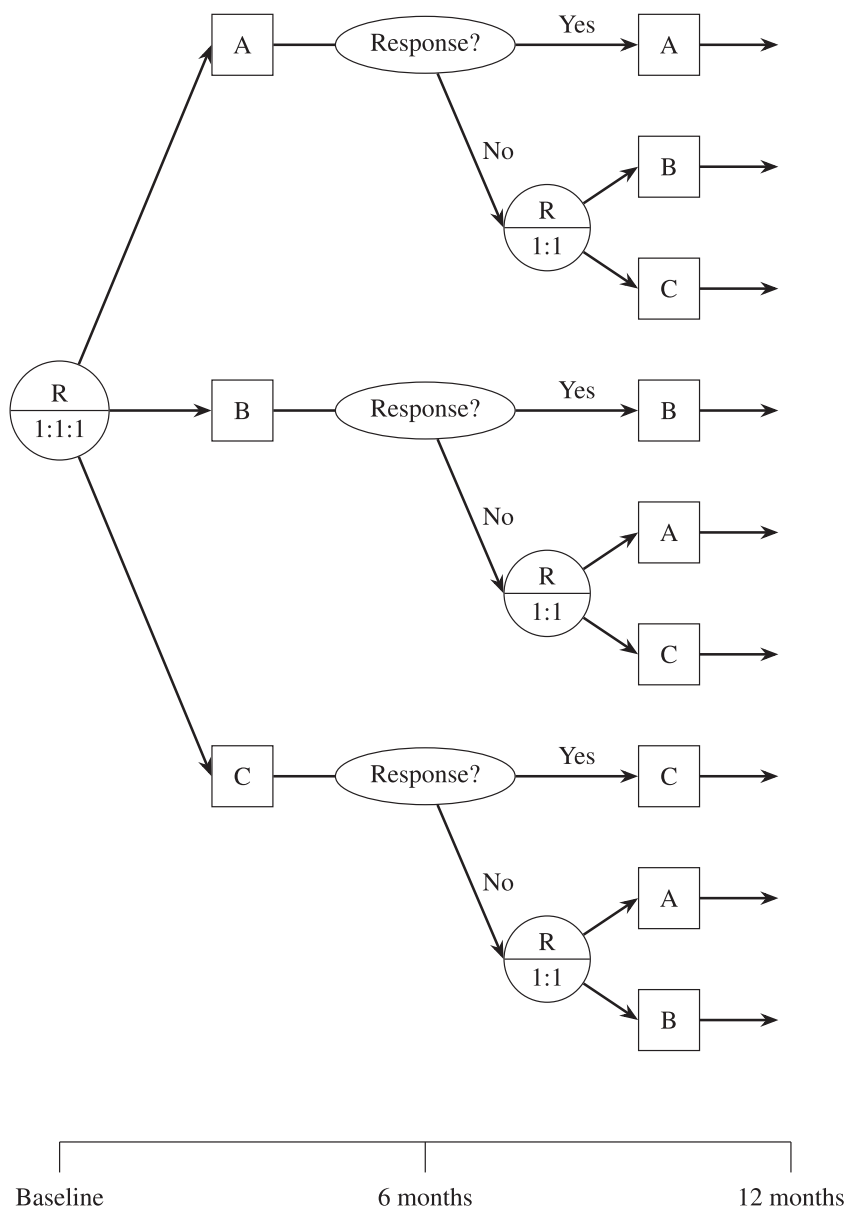

FIGURE 1 Study design of an snSMART. Patients are randomized (R) to one of the treatment arms, A, B or C equally (1:1:1) and followed for six months. The responders continue the same treatment for another six months, while the non-responders are re-randomized to one of the remaining treatments for an additional six months

This uncertainty is reflected in variation between providers, leading to patients being treated with agents of uncertain efficacy and unknown relative merit. ARAMIS compares the efficacy of three of the most commonly used treatments for the treatment of skin vasculitis, ie, colchicine, dapsone, and azathioprine (Figure 1). Eligible patients are randomized with equal chance of receiving one of the three treatments under investigation for six months. Those who do not respond after the first stage (ie, six months) are rerandomized equally between the other two treatments. Responders in stage 1 remain on their treatment in stage 2 . The outcome of interest is response to treatment at six months as defined by a combination of participant and physician measures.

In Section 2, we present a method to analyze data from an snSMART by sharing information across stages to evaluate the efficacy of each single treatment. The efficacy of a treatment is defined as the response rate at six months after initiating that treatment. In Section 3, we present simulation studies to illustrate our model's properties under various scenarios. Our paper concludes with a discussion in Section 4.

\section{METHOD}

The outcome of interest after each stage is a dichotomized variable, where 1 denotes response and 0 denotes nonresponse to the assigned treatment. We propose a Bayesian approach that borrows information across both stages to estimate the individual response rate of each treatment. The individual response rate we are interested in is a permanent feature of treatment similar to the treatment effect in a large parallel group trial. We model the first stage outcome as the probability of having a response to the first stage treatment. The second stage outcome is modeled conditionally on the first stage outcome linking the first and second stage response probabilities through linkage parameters. 
We compare the estimator of the response rate using the proposed method to estimators produced from three other methods, ie, (i) a log-Poisson model using data from both stages whose parameters are estimated via generalized estimating equations, (ii) a Bayesian method using only the first stage data, and (iii) a maximum likelihood method (maximum likelihood estimation) using only the first stage data. The details of our proposed model and the log-Poisson model will be discussed next, and simulation results for the comparison of estimators produced from the four methods will be shown in the Section 3.

\section{1 | Bayesian joint stage modeling}

For each subject $i=1, \ldots, N$, stage $j=1,2$, and treatment $k=A, B, \ldots K$, where $N$ denotes the total sample size and $K$ denotes the number of arms, let $Y_{i j k}$ denote the observed response outcome. We model the first stage outcome and the second stage outcome given the first stage outcome each as a Bernoulli random variable. The first stage response rate is denoted as $\pi_{k}$ for treatment $k$. The second stage response rate for first stage responders is equal to $\beta_{1} \pi_{k}$. For nonresponders to treatment $k$ in the first stage who receive treatment $k^{\prime}$ in the second stage, the second stage response rate in the second stage is equal to $\beta_{0} \pi_{k^{\prime}}$. In practice, we assume (i) the linkage parameters $\left(\beta_{0}, \beta_{1}\right)$ do not depend on the initial treatment $k$. (ii) The linkage parameter for nonresponders is smaller than 1 , ie, $\beta_{0}<1$. (iii) The linkage parameter for responders is greater than 1, ie, $\beta_{1}>1$. Via simulation, we examine the violations of the assumptions in Section 3.

Our proposed Bayesian joint stage model (BJSM) is as follows:

$$
\begin{gathered}
Y_{i 1 k} \mid \pi_{k} \backsim \operatorname{Bernoulli}\left(\pi_{k}\right) \\
Y_{i 2 k^{\prime}} \mid Y_{i 1 k}, \pi_{k} \backsim \operatorname{Bernoulli}\left(\left(\beta_{1} \pi_{k}\right)^{Y_{i 1 k}}\left(\beta_{0} \pi_{k^{\prime}}\right)^{1-Y_{i 1 k}}\right) .
\end{gathered}
$$

Prior distributions on the first stage response rates and the linkage parameters are used to incorporate physician beliefs about the treatments. For the ARAMIS trial, we specify priors for the parameters involved in the model $\pi_{k} \backsim B e t a\left(\zeta_{k}, \eta_{k}\right)$, $\beta_{0} \backsim \operatorname{Beta}\left(\zeta_{0}, \eta_{0}\right), \beta_{1} \backsim$ Pareto $(1, \phi)$. For $\pi_{k}$, we have chosen to use the hyperparameter values $\zeta_{k}=0.4$ and $\eta_{k}=1.6$ for two reasons. First, these parameters lead to a prior mean of $\zeta_{k} /\left(\zeta_{k}+\eta_{k}\right)=0.2$ for each of the arms, which was a reasonable a priori setting for the ARAMIS study. Second, the sum of the two parameters of a Beta distribution can be viewed as a prior sample size because the prior variance is inversely proportional to that sum. Thus, we assume our prior information is based upon a sample size of $\zeta_{k}+\eta_{k}=2$ patients. For $\beta_{0}$, we have chosen hyperparameter values $\zeta_{0}=1$ and $\eta_{0}=1$, which lead to a uniform distribution over the interval $[0,1]$. For $\beta_{1}$, we have assumed a hyperparameter value of $\phi=3$, so that, on average, the second stage response rate is $\phi /(\phi-1)=1.5$ times as large as the first stage response rate.

\section{2 | Log-Poisson joint stage modeling}

The log-Poisson joint stage model, which we refer to LPJSM, is a frequentist way of modeling data from two stages, where we use a log link to model the mean and the Poisson family to model the variance of the outcome. We model the the log of each response rate instead of the logit of each response rate mainly for interpretability. The model is as follows:

$$
\begin{aligned}
& \log \left(E\left(Y_{i 1 k}\right)\right)=\log \left(\mu_{i 1 k}\right)=\alpha_{A} \mathbb{1}\{k=A\}+\alpha_{B} \mathbb{1}\{k=B\}+\alpha_{C} \mathbb{1}\{k=C\} \\
& \log \left(E\left(Y_{i 2 k^{\prime}}\right)\right)=\log \left(\mu_{i 2 k^{\prime}}\right)=\alpha_{A} \mathbb{1}\left\{k^{\prime}=A\right\}+\alpha_{B} \mathbb{1}\left\{k^{\prime}=B\right\}+\alpha_{C} \mathbb{1}\left\{k^{\prime}=C\right\}+\gamma_{1} Y_{i 1 k}+\gamma_{0}\left(1-Y_{i 1 k}\right),
\end{aligned}
$$

where $\mathbb{1}\{\cdot\}$ is an indicator function. The response rates $\pi_{k}$ and the linkage parameters $\beta_{1}$ and $\beta_{0}$ from the BJSM are equivalent to the exponentiated values of $\alpha_{k}, \gamma_{1}$, and $\gamma_{0}$, respectively.

We estimate the parameters via generalized estimating equations ${ }^{18}$

$$
\sum_{i=1}^{N} \frac{\partial \boldsymbol{\mu}_{i}^{T}}{\partial \boldsymbol{\theta}} \boldsymbol{V}_{i}^{-1}\left(\boldsymbol{Y}_{i}-\boldsymbol{\mu}_{i}\right)=0,
$$

where $\boldsymbol{Y}_{i}=\left(Y_{i 1 k}, Y_{i 2 k^{\prime}}\right)^{T}, \boldsymbol{\mu}_{i}=\left(\mu_{i 1 k} ; \mu_{i 2 k^{\prime}}\right)^{T}, \boldsymbol{\theta}$ is the parameter vector with $\boldsymbol{\theta}=\left(\alpha_{A}, \alpha_{B}, \alpha_{C}, \gamma_{1}, \gamma_{0}\right)^{T}$, and $\boldsymbol{V}_{i}$ is the working covariance matrix of $\boldsymbol{Y}_{i}$ with $\boldsymbol{V}_{i}=\boldsymbol{A}_{i}^{1 / 2} \boldsymbol{R}(\alpha) \boldsymbol{A}_{i}^{1 / 2},{ }^{19}$ where $\boldsymbol{A}_{i}^{1 / 2}$ is a diagonal matrix with elements being the square root of $\operatorname{Var}\left(Y_{i j k}\right)$, the variance of the outcome of the $i$ th patient at the $j$ th stage under treatment $k$. The variance of the outcome of the $i$ th patient at the $j$ th stage is modeled with a Poisson family variance structure, $\operatorname{Var}\left(Y_{i j k}\right)=\mu_{i j k}$. We use the Poisson family variance structure to construct $V_{i}$ in Equation (3) to find the estimator of $\boldsymbol{\theta}$ as opposed to the binomial 
family variance structure because others have reported that estimation sometimes fails to converge when attempting to fit log-binomial models with a small sample size. ${ }^{20}$ In addition, we use an independence working correlation structure $\boldsymbol{R}(\alpha)=\mathbf{I}_{2 \times 2}$ in the estimating equation because the independence working correlation structure is recommended when binary responses have less than binomial variation over clusters. ${ }^{21}$

In estimating the variance of $\hat{\boldsymbol{\theta}}$, we use the robust "sandwich" covariance estimator, $\Sigma_{0}^{-1} \Sigma_{1} \Sigma_{0}^{-1}$, where $\Sigma_{0}=$ $\sum_{i=1}^{N} \frac{\partial \boldsymbol{\mu}_{i}^{T}}{\partial \boldsymbol{\theta}} \boldsymbol{V}_{i}^{-1} \frac{\partial \boldsymbol{\mu}_{i}^{T}}{\partial \boldsymbol{\theta}}$ and $\Sigma_{1}=\sum_{i=1}^{N} \frac{\partial \boldsymbol{\mu}_{i}^{T}}{\partial \boldsymbol{\theta}} \boldsymbol{V}_{i}^{-1}\left(\boldsymbol{Y}_{i}-\boldsymbol{\mu}_{i}\right)\left(\boldsymbol{Y}_{i}-\boldsymbol{\mu}_{i}\right)^{T} \boldsymbol{V}_{i}^{-1} \frac{\partial \boldsymbol{\mu}_{i}^{T}}{\partial \boldsymbol{\theta}}$. We use the binomial family variance structure, $\operatorname{Var}\left(Y_{i j k}\right)=\mu_{i j k}\left(1-\mu_{i j k}\right)$, to construct $\boldsymbol{V}_{i}$ in $\Sigma_{0}$ and $\Sigma_{1}$ to estimate variance of $\hat{\boldsymbol{\theta}}$ because the "sandwich" estimator is consistent, when $\boldsymbol{V}_{i}$ is correctly specified and even if $\boldsymbol{R}(\alpha)$ misspecified. ${ }^{22}$

\section{3 | SIMULATIONS}

We set up the scenarios for our simulation studies in two sections, ie, simulations when the assumptions for BJSM are satisfied and simulations when our assumptions are violated. We compare the bias, root-mean-square error (rMSE), coverage rate, and width of the $95 \%$ credible/confidence interval (CI) of the estimated parameters. When the BJSM assumptions are true, we compare the estimators from our proposed method (BJSM) to estimators produced from other three methods, ie, the LPJSM, described in Section 2.2, Bayesian first stage model (BFSM), and a first stage maximum likelihood estimates (FSMLEs). For both the BFSM and FSMLE, we only use the first stage data for estimation, and for the BFSM, we assume the same prior distribution for $\pi_{k}$ as the BJSM. When assumptions are violated, we compare the BJSM to an extension with multiple linkage parameters (BJSMM) and the LPJSM. The BJSMM is the same as that of the BJSM except that we allow the linkage parameters to depend on the initial treatment, ie, $\beta_{0}$ is now replaced by $\beta_{0 k}$ and $\beta_{1}$ is now replaced by $\beta_{1 k}$. We use exactly the same values for the hyperparameters as the BJSM for the prior densities of the BJSMM so that we allow for estimating $\beta_{0 k}$ and $\beta_{1 k}$ values that differ among different treatments $k$, but we give each the same prior distribution.

Bias is defined as the average of the differences between the true value of $\pi_{k}$ and the estimated $\pi_{k}$ in all simulations. The rMSE is calculated by taking the square root of the mean-square error of the estimators in all simulations. The simulated coverage rate is the frequency that the true value of the response paramter falls in the 95\% CI for all simulations. The 95\% CIs for the BJSM, BFSM, and BJSMM are the highest posterior density CIs, which is the narrowest interval that covers the $95 \%$ of the posterior distribution of $\pi_{k}$. The 95\% CIs for the LPJSM and FSMLE are derived based on the asymptotic normality of the estimator of $\pi_{k}$ in these two methods and calculated by the estimator plus or minus 1.96 times of the standard error of the estimator. The parameters are estimated via the $\mathrm{R}$ function jags and gee in the $\mathrm{R}$ package rjags and gee, respectively. The computer programs used to derive estimates and CIs are available upon request from the primary author.

\section{1 | Simulation scenarios}

We simulate 2000 realizations per scenario; each scenario is a three-arm snSMART. The true values of the response rates in each arm and the linkage parameters in each stage vary in different scenarios; details are presented in Table 1. We focus on simulation results where the total snSMART sample size is 90 (30 patients per treatment), but we provide results for total sample sizes of 45 and 180 in Section 6.

Scenarios 1 to 3 represent three ideal settings. In these scenarios, the linkage parameters for nonresponders and for responders are the same for all three treatments, which means the model specification is the same as the data generating process. In scenarios 4 to 12, we vary the values of the linkage parameters to investigate model properties when assumptions are violated (the assumptions are enumerated in Section 2.1). Assumption (i) is violated in scenarios 4 to 7 and 10 to 12 . Assumption (ii) is violated in scenarios 8,10 , and 12, and assumption (iii) is violated in scenarios 9,11 , and 12.

\subsection{Simulation results when the BJSM assumptions are true}

For scenarios 1 to 3, the bias and rMSE for estimators of the response rates are shown in Table 2. The response rate estimators of the BJSM have the smallest rMSEs among all four methods. The rMSEs of the estimators from the BJSM and LPJSM are smaller than the rMSEs provided by the BFSM and FSMLE, which only use data from the first stage. In scenario 2, the BJSM provides the estimators with smallest bias compared with other three methods. In scenarios 1 and 3, the bias of the estimators for the BJSM is still small but slightly higher than the bias for the other three methods. 
TABLE 1 Simulation scenarios. $\pi_{k}$ is the response rate at six months for the treatment

$k=A, B, C . \beta_{0 k}$ is the linkage parameter for the first stage nonresponders treated with treatment $k$. $\beta_{1 k}$ is the linkage parameter for the first stage responders treated with treatment $k$. The linkage parameters link the second stage response rates with the first stage response rate in our proposed model. The three assumptions are (i) the linkage parameters do not depend on the initial treatment $k$, ie, $\beta_{1 k}=\beta_{1}$ and $\beta_{0 k}=\beta_{0}$. (ii) The linkage parameter for nonresponders is smaller than 1 , ie, $\beta_{0}<1$. (iii) The linkage parameter for responders is greater than 1 , ie, $\beta_{1}>1$

\begin{tabular}{lcccccccccc} 
Scenarios & $\pi_{\boldsymbol{A}}$ & $\boldsymbol{\pi}_{\boldsymbol{B}}$ & $\boldsymbol{\pi}_{\boldsymbol{C}}$ & $\boldsymbol{\beta}_{\mathbf{0 A}}$ & $\boldsymbol{\beta}_{\mathbf{0} \boldsymbol{B}}$ & $\boldsymbol{\beta}_{\mathbf{0 C}}$ & $\boldsymbol{\beta}_{\mathbf{1 A}}$ & $\boldsymbol{\beta}_{\mathbf{1 B}}$ & $\boldsymbol{\beta}_{\mathbf{1 C}}$ & Assumptions Violated \\
1 & 0.3 & 0.3 & 0.3 & 0.8 & 0.8 & 0.8 & 1.5 & 1.5 & 1.5 & none \\
2 & 0.2 & 0.3 & 0.4 & 0.6 & 0.6 & 0.6 & 1.5 & 1.5 & 1.5 & none \\
3 & 0.2 & 0.3 & 0.4 & 0.8 & 0.8 & 0.8 & 1.5 & 1.5 & 1.5 & none \\
\hline 4 & 0.2 & 0.3 & 0.4 & 0.3 & 0.6 & 0.9 & 1.5 & 1.5 & 1.5 & (i) \\
5 & 0.2 & 0.3 & 0.4 & 0.6 & 0.6 & 0.6 & 1.2 & 1.5 & 1.8 & (i) \\
6 & 0.2 & 0.3 & 0.4 & 0.3 & 0.6 & 0.9 & 1.2 & 1.5 & 1.8 & (i) \\
7 & 0.2 & 0.3 & 0.4 & 0.9 & 0.6 & 0.3 & 1.2 & 1.5 & 1.8 & (i) \\
8 & 0.2 & 0.3 & 0.4 & 1.2 & 1.2 & 1.2 & 1.5 & 1.5 & 1.5 & (ii) \\
9 & 0.2 & 0.3 & 0.4 & 0.6 & 0.6 & 0.6 & 0.8 & 0.8 & 0.8 & (iii) \\
10 & 0.2 & 0.3 & 0.4 & 0.3 & 0.6 & 1.2 & 1.2 & 1.5 & 1.8 & (i), (ii) \\
11 & 0.2 & 0.3 & 0.4 & 1.2 & 1.2 & 1.2 & 0.8 & 0.8 & 0.8 & (ii), (iii) \\
12 & 0.2 & 0.3 & 0.4 & 0.3 & 0.6 & 1.2 & 0.8 & 1.5 & 1.8 & (i), (ii), (iii) \\
\hline
\end{tabular}

TABLE 2 Simulated bias and root-mean-square error (rMSE) for the estimators of $\pi_{k}$ where the Bayesian joint stage modeling (BJSM) assumptions are satisfied. Four modeling approaches, ie, BJSM, log-Poisson joint stage modeling (LPJSM), Bayesian first stage modeling (BFSM), and first stage maximum likelihood estimation (FSMLE) are compared. The sample size per treatment arm is $30 . \pi_{k}$ is the response rate at six months for treatment $k, k=A, B, C$

\begin{tabular}{lccccccccc} 
& & \multicolumn{2}{c}{ BJSM } & \multicolumn{2}{c}{ LPJSM } & \multicolumn{2}{c}{ BFSM } & \multicolumn{2}{c}{ FSMLE } \\
Scenario & & Bias & rMSE & Bias & rMSE & Bias & rMSE & Bias & rMSE \\
\hline 1 & & & & & & & & & \\
& $\pi_{A}$ & 0.008 & 0.062 & -0.001 & 0.069 & -0.008 & 0.079 & -0.002 & 0.084 \\
& $\pi_{B}$ & 0.008 & 0.062 & 0.002 & 0.069 & -0.006 & 0.078 & 0.001 & 0.083 \\
& $\pi_{C}$ & 0.008 & 0.061 & -0.002 & 0.068 & -0.008 & 0.078 & -0.001 & 0.083 \\
\hline & $\pi_{A}$ & -0.001 & 0.056 & -0.001 & 0.059 & -0.002 & 0.069 & -0.002 & 0.074 \\
& $\pi_{B}$ & 0.001 & 0.063 & 0.000 & 0.070 & -0.006 & 0.078 & 0.001 & 0.083 \\
& $\pi_{C}$ & 0.000 & 0.067 & 0.002 & 0.077 & -0.014 & 0.085 & -0.002 & 0.089 \\
\hline & $\pi_{A}$ & 0.005 & 0.056 & -0.001 & 0.057 & -0.002 & 0.069 & -0.002 & 0.074 \\
& $\pi_{B}$ & 0.008 & 0.062 & 0.000 & 0.069 & -0.006 & 0.078 & 0.001 & 0.083 \\
& $\pi_{C}$ & 0.011 & 0.064 & 0.002 & 0.076 & -0.014 & 0.085 & -0.002 & 0.089 \\
\hline
\end{tabular}

This may be because the prior mean for the linkage parameter for nonresponders is 0.5 , which is closer to 0.6 , the setting in scenario 2 , than 0.8 , the setting in scenarios 1 and 3 . These observations suggest that, in settings where the assumptions are satisfied, jointly modeling data from two stages provides improved estimators for treatment due to smaller rMSEs. In particular, the biggest gain in rMSE is given by the BJSM, which also produces small to negligible bias. Table 3 presents the $95 \%$ CI width and coverage rates. Here, we see the average width of the $95 \%$ CI of the BJSM is smaller than the other approaches, and the coverage rate is around the target $95 \%$.

\subsection{Simulation results when the BJSM assumptions are violated}

Simulation results when the assumptions for the BJSM are violated are shown in Tables 4 and 5. When only assumption (i) is violated (scenarios 4 to 7), we see that the bias for the response rate estimators is small for all three methods. The estimators of the BJSMM has the smallest bias in scenarios 4 to 6 and BJSM has the smallest bias in scenario 7. The estimators of the BJSM and BJSMM have smaller rMSEs than the LPJSM approach. When only assumption (ii) is violated, as in scenario 8, we see that the response rates are overestimated for all treatment arms by the BJSM and BJSMM. 
TABLE 3 Simulated width and coverage of 95\% CI for the estimators of $\pi_{k}$ where the Bayesian joint stage modeling (BJSM) assumptions are satisfied. Four modeling approaches, ie, BJSM,

log-Poisson joint stage modeling (LPJSM), Bayesian first stage modeling (BFSM), and first stage maximum likelihood estimation (FSMLE) are compared. The sample size per treatment arm is 30 . $\pi_{k}$ is the true response rate at six months for the treatment $k, k=A, B, C$. $\mathrm{CR}=$ Coverage Rate

\begin{tabular}{lccccccccc}
\multirow{2}{*}{ Scenario } & & \multicolumn{2}{c}{ BJSM } & \multicolumn{2}{c}{ LPJSM } & \multicolumn{2}{c}{ BFSM } & \multicolumn{2}{c}{ FSMLE } \\
\hline 1 & & Width & CR & Width & CR & Width & CR & Width & CR \\
& $\pi_{A}$ & 0.240 & 0.944 & 0.265 & 0.931 & 0.299 & 0.903 & 0.321 & 0.950 \\
& $\pi_{B}$ & 0.240 & 0.948 & 0.266 & 0.936 & 0.300 & 0.908 & 0.322 & 0.949 \\
& $\pi_{C}$ & 0.240 & 0.944 & 0.265 & 0.934 & 0.299 & 0.908 & 0.321 & 0.950 \\
\hline & $\pi_{A}$ & 0.213 & 0.929 & 0.228 & 0.932 & 0.256 & 0.945 & 0.277 & 0.945 \\
& $\pi_{B}$ & 0.245 & 0.940 & 0.269 & 0.936 & 0.300 & 0.908 & 0.322 & 0.949 \\
& $\pi_{C}$ & 0.265 & 0.948 & 0.305 & 0.937 & 0.323 & 0.930 & 0.344 & 0.930 \\
& $\pi_{A}$ & 0.210 & 0.936 & 0.222 & 0.936 & 0.256 & 0.945 & 0.277 & 0.945 \\
& $\pi_{B}$ & 0.240 & 0.942 & 0.263 & 0.936 & 0.300 & 0.908 & 0.322 & 0.949 \\
& $\pi_{C}$ & 0.258 & 0.956 & 0.300 & 0.937 & 0.323 & 0.930 & 0.344 & 0.930
\end{tabular}

TABLE 4 Simulated bias and root-mean-square error (rMSE) for the estimators of $\pi_{k}$ when assumptions are violated. Three modeling approaches, ie, Bayesian joint stage modeling (BJSM), BJSM to an extension with multiple linkage parameters (BJSMM), and log-Poisson joint stage modeling (LPJSM) are compared. The sample size per treatment arm is $30 . \pi_{k}$ is the response rate at six months for treatment $k, k=A, B, C$

\begin{tabular}{|c|c|c|c|c|c|c|c|}
\hline \multirow[b]{2}{*}{ Scenario } & & \multicolumn{2}{|c|}{ BJSM } & \multicolumn{2}{|c|}{ BJSMM } & \multicolumn{2}{|c|}{ LPJSM } \\
\hline & & Bias & rMSE & Bias & rMSE & Bias & rMSE \\
\hline \multirow[t]{3}{*}{4} & $\pi_{A}$ & -0.024 & 0.060 & -0.021 & 0.059 & -0.029 & 0.064 \\
\hline & $\pi_{B}$ & -0.004 & 0.063 & -0.001 & 0.062 & -0.007 & 0.070 \\
\hline & $\pi_{C}$ & 0.027 & 0.068 & 0.032 & 0.071 & 0.037 & 0.086 \\
\hline \multirow[t]{3}{*}{5} & $\pi_{A}$ & -0.010 & 0.054 & -0.005 & 0.055 & -0.011 & 0.056 \\
\hline & $\pi_{B}$ & -0.003 & 0.062 & 0.003 & 0.063 & -0.005 & 0.068 \\
\hline & $\pi_{C}$ & 0.022 & 0.072 & 0.017 & 0.070 & 0.017 & 0.080 \\
\hline \multirow[t]{3}{*}{6} & $\pi_{A}$ & -0.033 & 0.061 & -0.027 & 0.060 & -0.038 & 0.066 \\
\hline & $\pi_{B}$ & -0.008 & 0.062 & 0.000 & 0.062 & -0.012 & 0.069 \\
\hline & $\pi_{C}$ & 0.048 & 0.080 & 0.048 & 0.081 & 0.050 & 0.093 \\
\hline \multirow[t]{3}{*}{7} & $\pi_{A}$ & 0.014 & 0.055 & 0.017 & 0.058 & 0.017 & 0.059 \\
\hline & $\pi_{B}$ & 0.001 & 0.062 & 0.007 & 0.063 & 0.001 & 0.069 \\
\hline & $\pi_{C}$ & -0.004 & 0.073 & -0.013 & 0.073 & -0.018 & 0.080 \\
\hline \multirow[t]{3}{*}{8} & $\pi_{A}$ & 0.023 & 0.060 & 0.029 & 0.063 & -0.001 & 0.054 \\
\hline & $\pi_{B}$ & 0.036 & 0.069 & 0.042 & 0.073 & 0.000 & 0.065 \\
\hline & $\pi_{C}$ & 0.047 & 0.076 & 0.054 & 0.081 & 0.001 & 0.073 \\
\hline \multirow[t]{3}{*}{9} & $\pi_{A}$ & -0.015 & 0.054 & -0.016 & 0.054 & -0.001 & 0.061 \\
\hline & $\pi_{B}$ & -0.029 & 0.064 & -0.030 & 0.063 & 0.000 & 0.073 \\
\hline & $\pi_{C}$ & -0.047 & 0.075 & -0.047 & 0.075 & 0.002 & 0.081 \\
\hline \multirow[t]{3}{*}{10} & $\pi_{A}$ & -0.038 & 0.063 & -0.030 & 0.060 & -0.045 & 0.069 \\
\hline & $\pi_{B}$ & -0.015 & 0.063 & -0.005 & 0.062 & -0.024 & 0.070 \\
\hline & $\pi_{C}$ & 0.073 & 0.095 & 0.078 & 0.099 & 0.071 & 0.105 \\
\hline \multirow[t]{3}{*}{11} & $\pi_{A}$ & 0.011 & 0.053 & 0.014 & 0.054 & 0.000 & 0.056 \\
\hline & $\pi_{B}$ & 0.010 & 0.055 & 0.013 & 0.056 & 0.000 & 0.067 \\
\hline & $\pi_{C}$ & 0.006 & 0.055 & 0.010 & 0.055 & 0.001 & 0.076 \\
\hline \multirow[t]{3}{*}{12} & $\pi_{A}$ & -0.047 & 0.066 & -0.039 & 0.063 & -0.053 & 0.073 \\
\hline & $\pi_{B}$ & -0.014 & 0.063 & -0.005 & 0.062 & -0.022 & 0.070 \\
\hline & $\pi_{C}$ & 0.075 & 0.097 & 0.078 & 0.099 & 0.076 & 0.109 \\
\hline
\end{tabular}


TABLE 5 Simulated width and coverage of 95\% CI for the estimators of $\pi_{k}$ when assumptions are violated. Three modeling approaches: Bayesian joint stage modeling (BJSM), BJSM with multiple linkage parameters (BJSMM), and log-Poisson joint stage modeling (LPJSM) are compared. The sample size per treatment arm is $30 . \pi_{k}$ is the true response rate at six months for the treatment $k, k=A, B, C$. CR $=$ Coverage Rate

\begin{tabular}{|c|c|c|c|c|c|c|c|}
\hline \multirow[b]{2}{*}{ Scenario } & & \multicolumn{2}{|c|}{ BJSM } & \multicolumn{2}{|c|}{ BJSMM } & \multicolumn{2}{|c|}{ LPJSM } \\
\hline & & Width & CR & Width & CR & Width & CR \\
\hline \multirow[t]{3}{*}{4} & $\pi_{A}$ & 0.200 & 0.868 & 0.206 & 0.890 & 0.220 & 0.854 \\
\hline & $\pi_{B}$ & 0.240 & 0.933 & 0.247 & 0.948 & 0.266 & 0.929 \\
\hline & $\pi_{C}$ & 0.262 & 0.950 & 0.264 & 0.938 & 0.303 & 0.931 \\
\hline \multirow[t]{3}{*}{5} & $\pi_{A}$ & 0.208 & 0.932 & 0.213 & 0.936 & 0.215 & 0.915 \\
\hline & $\pi_{B}$ & 0.243 & 0.942 & 0.250 & 0.950 & 0.262 & 0.930 \\
\hline & $\pi_{C}$ & 0.270 & 0.942 & 0.278 & 0.951 & 0.311 & 0.942 \\
\hline \multirow[t]{3}{*}{6} & $\pi_{A}$ & 0.194 & 0.854 & 0.203 & 0.880 & 0.207 & 0.824 \\
\hline & $\pi_{B}$ & 0.238 & 0.936 & 0.248 & 0.946 & 0.259 & 0.923 \\
\hline & $\pi_{C}$ & 0.266 & 0.912 & 0.271 & 0.912 & 0.310 & 0.917 \\
\hline \multirow[t]{3}{*}{7} & $\pi_{A}$ & 0.220 & 0.957 & 0.223 & 0.949 & 0.222 & 0.948 \\
\hline & $\pi_{B}$ & 0.247 & 0.948 & 0.253 & 0.950 & 0.264 & 0.936 \\
\hline & $\pi_{C}$ & 0.272 & 0.928 & 0.284 & 0.935 & 0.313 & 0.930 \\
\hline \multirow[t]{3}{*}{8} & $\pi_{A}$ & 0.211 & 0.926 & 0.219 & 0.927 & 0.210 & 0.930 \\
\hline & $\pi_{B}$ & 0.235 & 0.922 & 0.245 & 0.922 & 0.252 & 0.938 \\
\hline & $\pi_{C}$ & 0.246 & 0.899 & 0.255 & 0.882 & 0.291 & 0.944 \\
\hline \multirow[t]{3}{*}{9} & $\pi_{A}$ & 0.206 & 0.926 & 0.207 & 0.933 & 0.238 & 0.934 \\
\hline & $\pi_{B}$ & 0.235 & 0.916 & 0.236 & 0.918 & 0.282 & 0.927 \\
\hline & $\pi_{C}$ & 0.250 & 0.892 & 0.251 & 0.900 & 0.316 & 0.932 \\
\hline \multirow[t]{3}{*}{10} & $\pi_{A}$ & 0.188 & 0.834 & 0.199 & 0.868 & 0.202 & 0.787 \\
\hline & $\pi_{B}$ & 0.232 & 0.922 & 0.244 & 0.942 & 0.255 & 0.902 \\
\hline & $\pi_{C}$ & 0.260 & 0.825 & 0.264 & 0.811 & 0.308 & 0.876 \\
\hline \multirow[t]{3}{*}{11} & $\pi_{A}$ & 0.207 & 0.949 & 0.212 & 0.950 & 0.217 & 0.928 \\
\hline & $\pi_{B}$ & 0.230 & 0.960 & 0.235 & 0.968 & 0.260 & 0.932 \\
\hline & $\pi_{C}$ & 0.238 & 0.972 & 0.243 & 0.974 & 0.298 & 0.944 \\
\hline \multirow[t]{3}{*}{12} & $\pi_{A}$ & 0.183 & 0.792 & 0.194 & 0.838 & 0.192 & 0.735 \\
\hline & $\pi_{B}$ & 0.232 & 0.925 & 0.244 & 0.942 & 0.258 & 0.907 \\
\hline & $\pi_{C}$ & 0.259 & 0.814 & 0.264 & 0.810 & 0.312 & 0.863 \\
\hline
\end{tabular}

The response rates are overestimated to balance the effect of the underestimated $\beta_{0}$ on the stage 2 response rates of nonresponders. The bias for the estimators is higher for BJSM and BJSMM but still small. The estimators from the LPJSM have the smallest bias and rMSEs. When only assumption (iii) is violated as in scenario 9 , the response rates are underestimated for all arms by the BJSM and BJSMM. The response rates are underestimated to balance the effect of the overestimated $\beta_{1}$ on the stage 2 response rates of responders. The estimators from the LPJSM have smallest bias but the rMSEs are higher compared with those from the BJSM and BJSMM.

When more than one assumption is violated (scenarios 10-12), the bias for the estimators of the response rates is lower in scenario 11, where assumption (i) holds and assumptions (ii) and (iii) are violated. This finding occurs because when assumption (ii) is violated, the BJSM and BJSMM tend to overestimate the response rates, and when assumption (iii) is violated, the BJSM and BJSMM tend to underestimate the response rates. and when both assumptions (ii) and (iii) are violated, two errors cancel each other. The BJSM and BJSMM have smaller rMSEs in all three scenarios.

In general, when the assumptions are violated, the response rate estimators of the BJSM have smaller bias and rMSEs than the LPJSM in most of the settings, and smaller rMSEs than the standard approaches that only use the data in stage 1. When multiple linkage parameters are considered in the BJSMM, we do not see a large reduction of bias or rMSEs.

In Table 5, we can see the average width of the 95\% CI of the BJSM is smaller than the other approaches. When only assumption (i) is violated (scenarios 4 to 7), the coverage rates of 95\% CIs for treatments B and C are around the target $95 \%$. The coverage rate is readily below the target for all three approaches in scenarios 4 and 6 . When only assumption (ii) (scenario 8) or assumption (iii) is violated (scenario 9), the coverage rate for the treatment $\mathrm{C}$ is below the target. 
This can be explained by the larger bias in the response rate estimator for treatment $C$ in scenarios 8 and 9. In scenario 10, (when assumption (i) and (ii) are violated), and scenario 12, when all the assumptions are violated, the coverage rates of 95\% CIs are below the target 95\% for treatment $\mathrm{A}$ and $\mathrm{C}$ because the response rate estimators have higher bias compared with the estimators of the treatment B. When assumptions (ii) and (iii) are violated at the same time, the coverage rates are greater than the target $95 \%$ for the BJSM and BJSMM and below the target 95\% for the LPJSM. Similar trends were observed for sample sizes of $n=45$ and 180; details are given in Tables 6-13 in Supporting Information.

\section{DISCUSSION}

In this manuscript, we have presented a Bayesian method (BJSM) to estimate the response rates of multiple treatments from snSMART with two stages. The BJSM is a novel method that links the response rates from two stages of one clinical trial via linkage parameters. The BJSM provides accurate estimators and straightforward clinical interpretations for the parameters. We compared the proposed method to three other methods via simulation and found that the BJSM provides the most accurate estimators among all four methods in small samples.

The BJSM relies on three key assumptions as outlined in Section 2.1. These assumptions simplify our model and make our model easier to interpret. However, there might be situations where the assumptions are violated. Simulation results suggest that the BJSM is able to estimate treatment arm response rates with small sample sizes even when the linkage parameters actually vary among the treatment arms. Nonetheless, further research is certainly warranted to determine the sample size at which moving from a BJSM to a BJSMM might be warranted.

Assumptions (ii) and (iii) in Section 2.1 that constrain the values of the linkage parameters generally hold when the response rates are similar and low (ie, less than 50\%) for all treatments in the trial. For many rare diseases, these assumptions are realistic, and thus, violations generally do not pose problems. If the assumptions are violated, the BJSM provides more biased estimates than the LPJSM but is more efficient. Sensitivity analyses using the LPJSM can always be done to compare results for the two methods.

A limitation that develops from our assumptions and corresponding priors is that the posterior distributions of $\beta_{1}$ and $\pi_{k}$ can have positive probability for $\beta_{1} \pi_{k}>1$. In reality, we can not have a response rate greater than 1 , but our models allow for this. To circumvent this potential problem, we considered a power model formulation of the BJSM. In the power model formulation, the second stage response rates are defined as $\pi_{k^{\prime}}^{\beta_{0}}$ and $\pi_{k}^{\beta_{1}}$ for nonresponders and responders, respectively. This allows $\beta_{0}$ and $\beta_{1}$ to vary on the positive real line. Ultimately, we decided against the power formulation as the linkage parameters are not clinically interpretable and our simulations for the proposed version of BJSM did not draw any samples such that $\beta_{1} \pi_{k}>1$, making it unlikely for this limitation to be a problem in practice in similar settings.

For ARAMIS, the binary six-month response is based on an objective measure (number of new lesions) that is further combined with two subjective measures, one from the physician and one from the patient. Thus, it seemed reasonable to collapse this complex set of outcomes into a single binary outcome of response. If ARAMIS had instead had a single objective continuous measure of efficacy, analysis of a dichotomized endpoint would have been unwise, due to the loss of information that dichotomization creates. We also emphasize that ARAMIS assumes no carry-over effects and no period effects. If carry-over effects and/or period effects exist in other applications, our proposed model would need to be modified.

Future work includes extending the BJSM to nonbinary outcomes (ie, continuous and survival outcomes) and establishing sample size calculations based on the analysis of snSMARTs using the BJSM. We aim to develop sample size calculations and an easy-to-use corresponding applet that can target specific differences between treatment arms. The sample size calculations will lead us to consider alternative designs of snSMARTs where more than three treatments are involved or there is an imbalance in second stage randomization. We are also working on another paper in which we extend our BJSM to estimate the treatment effects of dynamic treatment regimens.

\section{ACKNOWLEDGMENT}

This work was supported through a Patient-Centered Outcomes Research Institute (PCORI) Award (ME-1507-31108).

\section{ORCID}




\section{REFERENCES}

1. 107th Congress. Rare diseases act of 2002. Public Law 107-280. 2002.

2. Griggs RC, Batshaw M, Dunkle M, et al. Clinical research for rare disease: opportunities, challenges, and solutions. Mol Gen Metab. 2009;96:20-26.

3. Levin KA. Study design VII. Randomised controlled trials. Evid Based Dent. 2007;8:22-23.

4. Bell SA, Smith CT. A comparison of interventional clinical trials in rare versus non-rare diseases: an analysis of ClinicalTrials.gov. Orphanet J Rare Dis. 2014;9:1-11.

5. Gupta S, Faughnan ME, Tomlinson GA, Bayoumi AM. A framework for applying unfamiliar trial designs in studies of rare diseases. J Clin Epidemiol. 2011;64(10):1085-1094.

6. Evans SR. Clinical trial structures. J Exp Stroke Transl Med. 2010;3:8-18.

7. Makubate B, Senn S. Planning and analysis of cross-over trials in infertility. Statist Med. 2010;29(30):3203-3210.

8. Honkanen VEA, Siegel AF, Szalai JP, Berger V, Feldman BM, Siegel JN. A three-stage clinical trial design for rare disorders. Statist Med. 2001;20(20):3009-3021.

9. Tamura RN, Krischer JP, Pagnoux C, et al. A small $\mathrm{n}$ sequential multiple assignment randomized trial design for use in rare disease research. Contemp Clin Trials. 2016;46:48-51.

10. Lavori PW, Dawson R. A design for testing clinical strategies: biased adaptive within-subject randomization. $J R$ Stat Soc Ser A. 2000;163:29-38.

11. Murphy SA. An experimental design for the development of adaptive treatment strategies. Statist Med. 2005;24(10):1455-1481.

12. Dawson R, Lavori PW. Efficient design and inference for multistage randomized trials of individualized treatment policies. Biostatistics. 2011;13:142-152.

13. Robins J. A new approach to causal inference in mortality studies with a sustained exposure period - application to control of the healthy worker survivor effect. Math Model. 1986;7(9-12):1393-1512.

14. Murphy SA. Optimal dynamic treatment regimes. J R Stat Soc Stat Methodol Ser B. 2003;65(2):331-355.

15. Nason M, Follmann D. Design and analysis of crossover trials for absorbing binary endpoints. Biometrics. 2010;66(3):958-965.

16. Thall PF, Wooten LH, Logothetis CJ, Millikan RE, Tannir NM. Bayesian and frequentist two-stage treatment strategies based on sequential failure times subject to interval censoring. Statist Med. 2007;26(26):4687-4702.

17. Thall PF. Smart design, conduct, and analysis in oncology. In: Kosorok MR, Moodie EEM, Thall PF, eds. Adaptive Treatment Strategies in Practice: Planning Trials and Analyzing Data for Personalized Medicine. Philadelphia, PA: SIAM; 2016:41-54. ASA-SIAM Statistics and Applied Probablity Series.

18. Zeger SL, Liang K-Y. Longitudinal data analysis for discrete and continuous outcomes. Biometrics. 1986;42:121-130.

19. Pan W, Connett JE. Selecting the working correlation structure in generalized estimating equations with application to the lung health study. Stat Sin. 2002;12(2):475-490.

20. Williamson T, Eliasziw M, Fick GH. Log-binomial models: exploring failed convergence. Emerg Themes Epidemiol. 2013;10:1-10.

21. Hanley JA, Negassa A, Edwardes MD. GEE analysis of negatively correlated binary responses: a caution. Statist Med. 2000;19(5):715-722.

22. Halekoh U, Højsgaard S, Yan J. The R package geepack for generalized estimating equations. J Stat Softw. 2006;15(2):1-11.

\section{SUPPORTING INFORMATION}

Additional supporting information may be found online in the Supporting Information section at the end of the article.

How to cite this article: Wei B, Braun TM, Tamura RN, Kidwell KM. A Bayesian analysis of small $\mathrm{n}$ sequential multiple assignment randomized trials (snSMARTs). Statistics in Medicine. 2018;37:3723-3732. https://doi.org/10.1002/sim.7900 